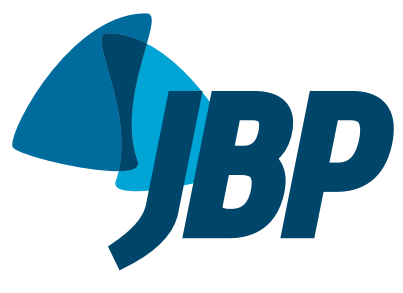

\title{
Pulmonary nontuberculous mycobacterial infections: presumptive diagnosis based on the international microbiological criteria adopted in the state of São Paulo, Brazil, 2011-2014
}

\author{
Lilian Regina Macelloni Marques ${ }^{1, a}$, Lucilaine Ferrazoli²,b Érica Chimara2,c
}

1. Núcleo de Ciências Biomédicas, Centro de Laboratório Regional, Instituto Adolfo Lutz de Marília, Marília (SP) Brasil.

2. Núcleo de Tuberculose e Micobacterioses, Centro de Bacteriologia, Instituto Adolfo Lutz, São Paulo (SP) Brasil.

a. (ID) http://orcid.org/0000-0002-6730-8099

b. (iD http://orcid.org/0000-0002-5389-7056

c. (D) http://orcid.org/0000-0001-9574-8449

Submitted: 12 September 2018. Accepted: 20 January 2019.

Study carried out at the Núcleo de Ciências Biomédicas, Centro de Laboratório Regional, Instituto Adolfo Lutz de Marilia, Marília (SP) Brasil and the Núcleo de Tuberculose e Micobacterioses, Centro de Bacteriologia, Instituto Adolfo Lutz, São Paulo (SP) Brasil.

\begin{abstract}
Objective: Pulmonary nontuberculous mycobacterial infections are caused by nontuberculous mycobacteria (NTM), the microbiological diagnosis of which involves the isolation and identification of the same species in at least two sputum samples, one BAL fluid sample, or one lung biopsy sample. The objective of the present study was to determine the frequency at which the various NTM species are identified among selected individuals and in potential cases of pulmonary nontuberculous mycobacterial infection. Methods: This was a retrospective analysis of the data on species isolated from respiratory specimens collected from 2,843 individuals between 2011 and 2014. Potential NTM infection cases were identified on the basis of the international microbiological criteria adopted in the state of São Paulo. Results: A total of 50 species were identified using the molecular method PCR-restriction enzyme analysis. Samples collected from 1,014 individuals were analyzed in relation to the microbiological criteria, and $448(44.18 \%)$ had a presumptive diagnosis of pulmonary nontuberculous mycobacterial infection, the species identified most frequently being, in descending order, Mycobacterium kansasii, M. abscessus, $M$. intracellulare, $M$. avium, and $M$. szulgai. Conclusions: Although various NTM species were identified among the individuals studied, those presumptively identified most frequently on the basis of the microbiological criteria adopted in the state of São Paulo were the ones that are most commonly associated with pulmonary nontuberculous mycobacterial infection worldwide or in specific geographic regions.

Keywords: Nontuberculous mycobacteria/classification; Mycobacterium infections, nontuberculous/diagnosis; Lung.
\end{abstract}

\section{INTRODUCTION}

Nontuberculous mycobacteria (NTM) are Mycobacterium spp. other than $M$. leprae and those that constitute the M. tuberculosis complex. NTM are widely distributed in nature, and some have been associated with human diseases that are characterized by pulmonary/ extrapulmonary manifestations that are collectively known as nontuberculous mycobacterial infections. In addition to differences in virulence among NTM, several host factors play a determinant role in the development of clinical manifestations of pulmonary nontuberculous mycobacterial infection, including structural lung changes caused by previous tuberculosis, cystic fibrosis, COPD, silicosis, and pneumoconiosis, as well as conditions that affect the immune system, such as alcoholism, smoking, and treatment with immunosuppressive drugs. ${ }^{(1-3)}$

The diagnosis of pulmonary nontuberculous mycobacterial infection is highly complex and requires a combination of clinical, radiological, and microbiological findings, as established by the American Thoracic Society/Infectious Diseases Society of America (ATS/IDSA) in 2007.(4) The clinical presentation of pulmonary nontuberculous mycobacterial infection is variable and nonspecific, and radiological features include cavitary and nodular lesions. The microbiological diagnosis of pulmonary nontuberculous mycobacterial infection involves the isolation and identification of the same NTM species in at least two sputum samples, one BAL fluid sample, or one lung biopsy sample. Although the ATS/IDSA did not specify the time interval between the first and second sputum sample collections in an official statement published in $2007,^{(4)}$ it had previously been reported that a 12-month interval is required for positive culture results. ${ }^{(5)}$

Because pulmonary nontuberculous mycobacterial infections are difficult to diagnose and because reporting is not compulsory, it is difficult to establish epidemiological markers; however, evidence suggests that the prevalence of pulmonary nontuberculous mycobacterial infections has steadily increased in recent years. ${ }^{(3,6)}$ The two NTM species that originally constituted the $M$. avium complex (MAC), namely $M$. avium and $M$. intracellulare, ${ }^{(7)}$ as well as $M$. abscessus, are the NTM species that are most commonly associated with pulmonary nontuberculous mycobacterial infection worldwide, whereas others appear 
to be more common in certain geographic regions. ${ }^{(3)}$ In Brazil, studies employing the ATS/IDSA criteria( ${ }^{(4)}$ have shown that the aforementioned species and $M$. kansasii are the most prevalent causes of pulmonary nontuberculous mycobacterial infection in the states of Rio de Janeiro ${ }^{(8)}$ and Rio Grande do Sul. ${ }^{(9)}$

The frequency at which the various NTM species are identified in individuals with pulmonary nontuberculous mycobacterial infection has been determined on the basis of the ATS/IDSA microbiological criteria alone. ${ }^{(10-12)}$ The present study is a retrospective analysis of the data on NTM species isolated from respiratory specimens in a referral laboratory in the state of São Paulo, Brazil, between 2011 and 2014. Our objective was to determine the frequency at which the various NTM species are identified among selected individuals and in potential cases of pulmonary nontuberculous mycobacterial infection on the basis of microbiological criteria.

\section{METHODS}

The Instituto Adolfo Lutz (IAL, Adolfo Lutz Institute) is a referral laboratory in the state of São Paulo. In addition to the IAL headquarters (a referral center for tuberculosis and nontuberculous mycobacterial infections in the city of São Paulo, Brazil), there are twelve regional laboratories strategically located throughout the state of São Paulo. Mycobacterial cultures are performed in the regional laboratories and in approximately 80 others (either public laboratories or laboratories affiliated with the Brazilian Unified Health Care System), and all positive cultures are sent to the IAL headquarters for identification. All patient data provided by the regional laboratories and the results of isolate identification are recorded in a laboratory information management system (LIMS). For the present study, the results of isolate identification for the period between 2011 and 2014 were entered into Microsoft Excel 2007 spreadsheets (one for each year), data on NTM species isolated from respiratory specimens being subsequently selected and entered into a new Microsoft Excel 2007 spreadsheet.

In the new spreadsheet, the first record of each individual was selected in order to identify patients for inclusion in the study ("selected patients"), and new spreadsheets were created for each NTM species identified during the study period. Patient laboratory records were reviewed in order to identify patients meeting the requirements for analysis of the ATS/IDSA microbiological criteria for the diagnosis of pulmonary nontuberculous mycobacterial infection, ${ }^{(4)}$ all data being entered into the aforementioned spreadsheets. Subsequently, spreadsheets were created for those who met the requirements for analysis of the ATS/ IDSA diagnostic criteria ("analyzed patients") and those who had a presumptive diagnosis based on the microbiological criteria ("identified patients") for each NTM isolate.

All NTM species were identified by PCR-restriction enzyme analysis (PRA), which consists of amplification of a 441-bp fragment of the hsp65 gene, followed by digestion of the amplified product with two restriction enzymes (BstEII and HaeIII) and analysis of the fragments separated by agarose gel electrophoresis. The various NTM species have different restriction patterns, which can be used for species identification by means of an algorithm. ${ }^{(13)}$

The present study was approved by the Technical and Scientific Advisory Committee and the Research Ethics Committee of the IAL (CTC no. 07-G/2014).

\section{RESULTS}

Between 2011 and 2014, a total of 5,392 NTM were isolated from respiratory specimens from a total of 3,883 individuals. The hsp65 PRA method failed to identify the NTM species in 881 patients (22.69\%), isolates being phenotypically characterized as slowly growing mycobacteria ( $n=630$ ) or rapidly growing mycobacteria $(n=251)$. A restriction pattern suggestive of mixed culture (i.e., indicative of the presence of NTM and $M$. tuberculosis complex) was found in samples from 159 patients $(4.09 \%)$. Therefore, 2,843 patients were selected for inclusion in the study.

Of the 2,843 patients selected for inclusion in the study, only $1,014(35.67 \%)$ met the requirements for analysis of the ATS/IDSA microbiological criteria ${ }^{(4)}$ for the diagnosis of pulmonary nontuberculous mycobacterial infection. A total of 50 species were identified, and Table 1 shows the frequency at which they were identified among selected patients and analyzed patients. In both groups of patients, 6 NTM species were most common, the proportions for analyzed/selected patients being as follows, in descending order: M. abscessus, M. kansasii, M. avium, M. fortuitum, M. intracellulare, and $M$. gordonae.

As can be seen in Table 2, 448 (44.18\%) of the 1,014 patients who were analyzed in relation to the microbiological criteria were identified as potential cases of pulmonary nontuberculous mycobacterial infection. The remaining 566 had one culture with NTM growth and, subsequently, no mycobacterial growth on culture. Among the patients who were identified as potential cases of pulmonary nontuberculous mycobacterial infection, the NTM species identified most frequently were M. kansasii (66.50\%) and M. abscessus (64.71\%), followed by $M$. intracellulare $(60.69 \%)$ and $M$. avium (46.97\%). M. gordonae and $M$. fortuitum were found in a considerable number of analyzed patients. However, among those who were identified as potential cases of pulmonary nontuberculous mycobacterial infection, they were found in only $20.62 \%$ and $13.68 \%$, respectively.

A total of 19 NTM species were identified among the 568 patients who had only one positive culture (the others being negative) and who therefore were not presumptively diagnosed with pulmonary nontuberculous mycobacterial infection. The 19 NTM species identified were as follows: M. asiaticum, M. chitae, M. flavescens, M. florentinum, M. gastri, M. genavense, M. goodii, $M$. heidelbergense, M. immunogenum, M. kumamotonense, 
Table 1. Frequency and proportion of nontuberculous mycobacterial species among the patients selected for the study and those analyzed in relation to the microbiological criteria for the diagnosis of pulmonary nontuberculous mycobacterial infections in the state of São Paulo, Brazil, 2011-2014.

\begin{tabular}{|c|c|c|c|}
\hline \multirow[t]{2}{*}{ Species } & \multicolumn{2}{|c|}{ Patients, n (\%) } & \multirow{2}{*}{$\begin{array}{c}\text { Analyzed/selected ratio, } \\
\%\end{array}$} \\
\hline & $\begin{array}{c}\text { Selected } \\
\text { (n }=2,843)\end{array}$ & $\begin{array}{c}\text { Analyzed } \\
(n=1,014)\end{array}$ & \\
\hline Mycobacterium avium & $519(18.26)$ & $198(19.53)$ & 38.15 \\
\hline M. kansasii & $453(15.93)$ & $197(19.43)$ & 43.49 \\
\hline M. intracellulare & $423(14.88)$ & $145(14.30)$ & 34.28 \\
\hline M. gordonae & $374(13.16)$ & $97(9.57)$ & 25.94 \\
\hline M. fortuitum & $310(10.90)$ & $117(11.54)$ & 37.74 \\
\hline M. abscessus & $219(7.70)$ & $119(11.74)$ & 54.34 \\
\hline M. peregrinum & $143(5.03)$ & $35(3.45)$ & 24.48 \\
\hline M. chelonae & $96(3.38)$ & $19(1.87)$ & 19.79 \\
\hline M. mucogenicum & $66(2.32)$ & $16(1.58)$ & 24.24 \\
\hline M. lentiflavum & $46(1.62)$ & $9(0.89)$ & 19.57 \\
\hline M. simiae & $26(0.91)$ & $7(0.69)$ & 26.92 \\
\hline M. szulgai & $19(0.67)$ & $11(1.08)$ & 57.89 \\
\hline M. asiaticum & $16(0.56)$ & $6(0.59)$ & 37.50 \\
\hline M. florentinum & $15(0.53)$ & $1(0.10)$ & 6.67 \\
\hline M. chitae & $14(0.49)$ & $4(0.39)$ & 28.57 \\
\hline M. goodii & $14(0.49)$ & $5(0.49)$ & 35.71 \\
\hline M. nonchromogenicum & $12(0.42)$ & $6(0.59)$ & 50.00 \\
\hline M. neoaurum & $8(0.28)$ & $2(0.20)$ & 25.00 \\
\hline M. parascrofulaceum & $8(0.28)$ & $2(0.20)$ & 25.00 \\
\hline M. novocastrense & $7(0.25)$ & $1(0.10)$ & 14.29 \\
\hline M. xenopi & $6(0.21)$ & $3(0.30)$ & 50.00 \\
\hline M. terrae & $5(0.18)$ & $1(0.10)$ & 20.00 \\
\hline M. immunogenum & $4(0.14)$ & $1(0.10)$ & 25.00 \\
\hline M. heidelbergense & $3(0.11)$ & $1(0.10)$ & 33.33 \\
\hline M. kumamotonense & $3(0.11)$ & $1(0.10)$ & 33.33 \\
\hline M. nebraskense & $3(0.11)$ & $3(0.30)$ & 100.00 \\
\hline M. celatum & $2(0.07)$ & $1(0.10)$ & 50.00 \\
\hline M. flavescens & $2(0.07)$ & $1(0.10)$ & 50.00 \\
\hline M. gastri & $2(0.07)$ & $1(0.10)$ & 50.00 \\
\hline M. parmense & $2(0.07)$ & $1(0.10)$ & 50.00 \\
\hline M. sherrisii & $2(0.07)$ & $1(0.10)$ & 50.00 \\
\hline M. brisbanense & $2(0.07)$ & 0 & - \\
\hline M. monacense & $2(0.07)$ & 0 & - \\
\hline M. triviale & $2(0.07)$ & 0 & - \\
\hline M. genavense & $1(0.04)$ & $1(0.10)$ & 100.00 \\
\hline M. triplex & $1(0.04)$ & $1(0.10)$ & 100.00 \\
\hline Others $^{\mathrm{a}}$ & $13(0.46)$ & 0 & - \\
\hline
\end{tabular}

aThirteen species for which only one patient was selected: M. arupense, M. aubagnense, M. branderi, M. brumae, M. conceptionense, M. farcinogenes, M. hiberniae, M. kubicae, M. montefiorense, M. scrofulaceum, M. senegalense, M. shimoidei, and M. wolinskyi.

M. mucogenicum, M. nebraskense, M. neoaurum, M. nonchromogenicum, M. novocastrense, M. parmense, M. terrae, M. triplex, and M. xenopi.

\section{DISCUSSION}

The identification of NTM species is extremely important because the clinical relevance and antimicrobial treatment of pulmonary nontuberculous mycobacterial infections are determined on the basis of the characteristics of the NTM species. Although the molecular method used in the present study (i.e., the hsp65 PRA method) is more rapid and specific than phenotypic methods, some of its limitations include the fact that PCR inhibitors interfere with the sample and the fact that the available algorithm does not define the restriction patterns of the species. In the present study, the hsp65 PRA method failed to identify the NTM isolates from $22.68 \%$ of the selected patients, the species being phenotypically characterized. Esparcia et al. ${ }^{(14)}$ used the same algorithm that was used in the 
Table 2. Species of nontuberculous mycobacteria isolated from respiratory specimens from patients analyzed in relation to microbiological criteria for the diagnosis of pulmonary nontuberculous mycobacterial infections, as well as frequency of patients identified as potential cases of pulmonary nontuberculous mycobacterial infection in the state of São Paulo, Brazil, 2011-2014.

\begin{tabular}{|c|c|c|c|}
\hline \multirow[t]{2}{*}{ Species } & \multicolumn{3}{|c|}{ Number of patients } \\
\hline & Analyzed & Identified & $\%$ \\
\hline $\begin{array}{l}\text { Mycobacterium } \\
\text { kansasii }\end{array}$ & 197 & 131 & 66.50 \\
\hline M. abscessus & 119 & 77 & 64.71 \\
\hline M. intracellulare & 145 & 88 & 60.69 \\
\hline M. avium & 198 & 93 & 46.97 \\
\hline M. szulgai & 11 & 5 & 45.45 \\
\hline M. peregrinum & 35 & 8 & 22.86 \\
\hline M. chelonae & 19 & 4 & 21.05 \\
\hline M. gordonae & 97 & 20 & 20.62 \\
\hline M. goodii & 5 & 1 & 20.00 \\
\hline M. simiae & 7 & 1 & 14.29 \\
\hline M. fortuitum & 117 & 16 & 13.68 \\
\hline M. lentiflavum & 9 & 1 & 11.11 \\
\hline Others $^{\mathrm{a}}$ & 55 & $3^{\mathrm{b}}$ & 5.45 \\
\hline Total & 1,014 & 448 & 44.18 \\
\hline
\end{tabular}

aTwenty-one species (for 15 , only 1 or 2 patients were analyzed). ${ }^{\mathrm{b}} \mathrm{M}$. celatum, M. parascrofulaceum, and $M$. sherrisii.

present study and reported that the hsp65 PRA method failed to identify 32 (23.88\%) of 134 NTM samples.

Although a wide variety of NTM species were identified in the present study, the most frequently identified species among selected and analyzed patients were, in descending order, M. avium, M. kansasii, $M$. intracellulare, $M$. gordonae, $M$. fortuitum, and $M$. abscessus. In a study conducted in the state of Rio Grande do Sul in the 2003-2013 period, the most frequently identified species were $M$. avium, M. kansasii, and $M$. intracellulare, followed by $M$. abscessus, $M$. fortuitum, and M. gordonae. ${ }^{(9)}$ Between 1993 and 2011 in the state of Rio de Janeiro, the most frequently identified species was M. kansasii, followed by MAC, M. abscessus, and M. fortuitum. ${ }^{\left({ }^{8}\right)}$ It is of note that $M$. abscessus was the most frequently identified species among analyzed patients in the present study. This might be due to the fact that $M$. abscessus is resistant to many antimicrobial agents ${ }^{(3)}-$ a fact that impedes patient clinical improvement-as well as to the fact that health professionals have limited knowledge of the relevance of $M$. abscessus.

Less than $40 \%$ of the selected patients in the present study met the requirements for analysis of the microbiological criteria for the diagnosis of pulmonary nontuberculous mycobacterial infection. This is probably due to the fact that the clinical specimens obtained after NTM identification were examined in laboratories where the IAL-LIMS is not used, screening therefore being impossible when the results were negative. However, in many patients for whom NTM cultures were performed in laboratories where the IAL-LIMS is used, no subsequent specimens were sent for analysis. This is probably due to loss to follow-up and a lack of knowledge on the part of the health care team regarding the need for multiple sputum examinations for laboratory diagnosis of pulmonary nontuberculous mycobacterial infection.

Given that NTM are widely distributed in nature, the isolation of NTM from a clinical specimen can represent colonization, infection, or pseudoinfection. Colonization is defined as the presence of NTM in the host microbiota without clinical manifestations, whereas infection is defined as the presence of NTM in the host microbiota with clinical manifestations. When neither colonization nor infection can be unequivocally confirmed, the possibility of pseudoinfection should be considered, pseudoinfection being usually caused by contamination during clinical specimen handling. There have been reports of outbreaks of nosocomial colonization and pseudoinfection with NTM primarily due to contaminated potable water supplies and inadequately disinfected medical equipment. ${ }^{(15)}$

In the present study, 14 NTM species led to a presumptive diagnosis of pulmonary nontuberculous mycobacterial infection based on microbiological criteria. The number of analyzed patients and the proportion of patients identified as potential cases of pulmonary nontuberculous mycobacterial infection were highest for the NTM species on which the ATS/IDSA criteria were based, (4) i.e., M. avium/M. intracellulare (MAC), M. kansasii, and M. abscessus. As in the present study, $M$. fortuitum, M. peregrinum, M. chelonae, and $M$. szulgai led to a presumptive diagnosis of pulmonary nontuberculous mycobacterial infection in a study conducted in Japan and employing microbiological criteria. ${ }^{(12)}$ The fact that individuals with one positive culture $^{(16)}$ or more than one positive culture(17) for $M$. fortuitum were monitored for months and did not progress to lung disease was attributed to the fact that M. fortuitum is a low-virulence organism. M. gordonae is also considered to be a low-virulence strain and has been associated with several outbreaks of nosocomial mycobacterial pseudoinfection. ${ }^{(18)}$

Winthrop et al.(19) found that 183 (86\%) of 214 patients with a presumptive diagnosis of pulmonary nontuberculous mycobacterial infection based on the ATS/IDSA microbiological criteria( ${ }^{(4)}$ had a confirmed diagnosis of pulmonary nontuberculous mycobacterial infection after analysis of clinical and radiological features, the authors having concluded that the ATS/ IDSA microbiological criteria( ${ }^{(4)}$ are highly predictive of pulmonary nontuberculous mycobacterial infection. According to the ATS/IDSA, patients who are suspected of having pulmonary nontuberculous mycobacterial infection but do not meet the diagnostic criteria should be monitored until the diagnosis is firmly established or excluded. ${ }^{(4)}$ Extensive clinical and laboratory monitoring of NTM isolates from clinical specimens obtained from patients with respiratory symptoms allows assessment of the relevance of NTM species, especially with regard to potential risk factors. 
To our knowledge, ours is the first study in Brazil to determine the frequency at which the various NTM species are identified in a large number of individuals in a large geographic area rather than in a large number of respiratory specimens. The present study has limitations inherent to all retrospective studies, including missing information on patient medication use when multiple cultures are performed, the lack of which is minimized by innate NTM resistance to antimicrobials. Although various NTM species were identified in the individuals studied, those presumptively identified most frequently on the basis of the international microbiological criteria used in the state of São Paulo were the ones that are most commonly associated with pulmonary nontuberculous mycobacterial infection worldwide or in specific geographic regions. Health professionals should know more about pulmonary nontuberculous mycobacterial infections. There is a need for prospective multidisciplinary studies in which all of the diagnostic criteria established by the ATS/ IDSA $^{(4)}$ are used and all of the characteristics of study participants are determined.

\section{REFERENCES}

1. Katoch VM. Infections due to non-tuberculous mycobacteria (NTM). Indian J Med Res. 2004;120(4):290-304

2. Weiss $\mathrm{CH}$, Glassroth J. Pulmonary disease caused by nontuberculous mycobacteria. Expert Rev Respir Med. 2012;6(6):597-612; quiz 613. https://doi.org/10.1586/ers.12.58

3. Stout JE, Koh W, Yew WW. Update on pulmonary disease due to non-tuberculous mycobacteria. Int J Infect Dis. 2016;45:123-34. https://doi.org/10.1016/j.ijid.2016.03.006

4. Griffith DE, Aksamit T, Brown-Elliott BA, Catanzaro A, Daley C, Gordin F, et al. An official ATS/IDSA statement: diagnosis, treatment, and prevention of nontuberculous mycobacterial diseases. Am J Respir Crit Care Med. 2007;175(4):367-416. https://doi.org/10.1164/ rccm.200604-571ST

5. Diagnosis and treatment of disease caused by nontuberculous mycobacteria. This official statement of the American Thoracic Society was approved by the Board of Directors, March 1997. Medical Section of the American Lung Association. Am J Respir Crit Care Med. 1997;156(2 Pt 2):S1-25.

6. Prevots DR, Marras TK. Epidemiology of human pulmonary infection with non-tuberculous mycobacteria: a review. Clin Chest Med. 2015;36(1):13-34. https://doi.org/10.1016/j.ccm.2014.10.002

7. Kim SY, Shin SH, Moon SM, Yang B, Kim H, Kwon OJ, et al. Distribution and clinical significance of Mycobacterium avium complex species isolated from respiratory specimens. Diagn Microbiol Infect Dis. 2017;88(2):125-137. https://doi.org/10.1016/j. diagmicrobio.2017.02.017

8. de Mello KG, Mello FC, Borga L, Rolla V, Duarte RS, Sampaio EP, et al. Clinical and therapeutic features of pulmonary nontuberculous mycobacterial disease, Brazil, 1993-2011. Emerg Infect Dis. 2013;19(3):393-9.

9. Carneiro MDS, Nunes LS, David SMM, Dias CF, Barth AL, Unis G. Nontuberculous mycobacterial lung disease in a high tuberculosis incidence setting in Brazil. J Bras Pneumol. 2018;44(2):106-111. https://doi.org/10.1590/s1806-37562017000000213

10. Prevots DR, Shaw PA, Strickland D, Jackson LA, Raebel MA, Blosky $M A$, et al. Nontuberculous mycobacterial lung disease prevalence at four integrated health care delivery systems. Am J Respir Crit Care Med. 2010;182(7):970-6. https://doi.org/10.1164/rccm.20100203100C

11. Henkle E, Hedberg K, Schafer S, Novosad S, Winthrop KL.
Population-based Incidence of Pulmonary Nontuberculous Mycobacterial Disease in Oregon 2007 to 2012. Ann Am Thorac Soc. 2015;12(5):642-7. https://doi.org/10.1513/AnnalsATS.201412-5590C

12. Morimoto $K$, Hasegawa $N$, Izumi $K$, Namkoong $H$, Uchimura $K$, Yoshiyama T, et al. A Laboratory-based Analysis of Nontuberculous Mycobacterial Lung Disease in Japan from 2012 to 2013. Ann Am Thorac Soc. 2017;14(1):49-56. https://doi.org/10.1513/ AnnalsATS.201607-5730C

13. Chimara E, Ferrazoli L, Ueky SY, Martins MC, Durham AM, Arbeit $R D$, et al. Reliable identification of mycobacterial species by PCRrestriction enzyme analysis (PRA)-hsp65 in a reference laboratory and elaboration of a sequence-based extended algorithm of PRA-hsp65 patterns. BMC Microbiol. 2008;8:48. https://doi.org/10.1186/14712180-8-48

14. Esparcia Ó, Español M, Garrigó M, Moreno C, Montemayor M, Navarro F, et al. Use of different PCR-based techniques integrated into a non-tuberculous identification algorithm [Article in Spanish]. Enferm Infecc Microbiol Clin. 2012;30(1):3-10. https://doi. org/10.1016/j.eimc.2011.06.014

15. Phillips $M$, von Reyn CF. Nosocomial infections due to nontuberculous mycobacteria. Clin Infect Dis. 2001;33(8):1363-74 https://doi.org/10.1086/323126

16. Lee MR, Yang Cy, Shu CC, Lin CK, Wen YF, Lee SW, et al. Factors associated with subsequent nontuberculous mycobacterial lung disease in patients with a single sputum isolate on initial examination. Clin Microbiol Infect. 2015;21(3):250.e1-e7. https://doi.org/10.1016/j. cmi.2014.08.025

17. Park S, Suh GY, Chung MP, Kim H, Kwon OJ, Lee KS, et al. Clinical significance of Mycobacterium fortuitum isolated from respiratory specimens. Respir Med. 2008;102(3):437-42. https://doi. org/10.1016/j.rmed.2007.10.005

18. Scorzolini L, Mengoni F, Mastroianni CM, Baldan R, Cirillo DM, De Giusti $M$, et al. Pseudo-outbreak of Mycobacterium gordonae in a teaching hospital: importance of strictly following decontamination procedures and emerging issues concerning sterilization. New Microbiol. 2016;39(1):25-34.

19. Winthrop KL, McNelley E, Kendall B, Marshall-Olson A, Morris $C$, Cassidy $M$, et al. Pulmonary nontuberculous mycobacteria disease prevalence and clinical features: an emerging public health disease. Am J Respir Crit Care Med. 2010;182(7):977-82. https://doi. org/10.1164/rccm.201003-05030C 